\title{
Crack identification for transient heat operator by using domain decomposition method
}

\author{
Anis Bel Hadj Hassin and Sinda Khalfallah \\ Université de Tunis El Manar, Ecole Nationale d’Ingénieurs de Tunis, LR99ES20 Modèlisation Mathématique et Numérique dans les \\ Sciences de L'ingénieurs, LAMSIN, B.P. 37, 1002 Tunis, Tunisie
}

Received: 26 March 2017, Accepted: 19 June 2017

Published online: 25 August 2017.

\begin{abstract}
This work deals with cracks identification from over-determined boundary data. The consideration physical phenomena corresponds to the transient heat equation. we give a theoretical result of identifiability for the inverse problem under consideration. Then, we consider a recovering process based on coupling domain decomposition method and minimizing an energy-type error functional. The efficiency of the proposed approach is illustrated by several numerical results.
\end{abstract}

Keywords: Inverse problem, crack, identifiability, transient heat equation, ill-posed problem, Cauchy problem, domain decomposition, virtual control, noise.

\section{Introduction}

Various works tackled the problem of the detection of interior cracks in a given physical body from abundant boundary data. The involved physical can be either thermal, electrostatica, acoustical or elastostatital. Such a problem arises in different engineering fields such as non-destructive testing of materials. Up to our knowledge, there are very few quasi-explicit methods. These methods does not allow the recovring the crack, they give only qualitative results (information on the shape and the location of the crack). Further more, they are usually based on the essential assumption that the over determined boundary data are 'complete', which means that they are known on the whole outer boundary of the body (Andrieux and Ben Abda 1996 [4]; Brühl et al. 2001 [14]; Baratchart et al. 1999 [7]).

Typically, the identification of cracks is an ill-posed inverse problem, in the sense of Hadamard [18]. Theoretical results on identiÂfiability of cracks are seldom and mainly focus on the Laplace equation. In [16], Friedman and Vogelius proved that two appropriate current fluxes, together with the corresponding voltages, are necessary and suffice to uniquely determine a single crack. Bryan and Volgelius [15] , followed by Alessandrini and Diaz Valenzuela [1] examined the multiple crack problem always in 2D situation.

In the case of the transient heat equation, A. Ben Abda and H. D. Bui [9] proposed a theoretical technique of the identification of planar crack based on the reciprocity gap concept.

Due to the ill-posedness character of the problem of cracks identification, there is a growing need for specified computational methods for their numerical resolution. The aim of this work is to solve the problem of identification of cracks in transient heat problem, from the measurement on outer edge, by the domain decomposition method (DDM) and virtual control. The approach that we propose consists in using the DDM method in order to transform the problem of crack identification into two boundary inverse problems. The later are them selfs classical ill-posed problems and there 
are several works which are dedicated for their numerical treatment $[8,12,28]$. Classical approaches using a least-squares formulation were proposed $[11,13,25]$ and which require some regularization tool to solve these ill-posed problems.

Here, for each boundary inverse problem, we consider an approach based on an energy norm defined from two well-posed problems, which appears to be self-regularizing [2,3]. There are several ways to minimize an energy norm which use a least-squares formulation. We extend the method introduced in $[19,23]$ for the steady-state case, whereas we consider here a transient case. The idea is to write the first-order optimality conditions as an interface interfacial problem [11]. After that, we apply a preconditioned iterative gradient algorithm, at each time step, [24], which appears to be very efficient.

This paper is organized as follows. In section 2, the forward problem is presented. Section 3 is devoted to the identifiability result from one single measurement. In Section 4, we introduce the method of the fictitious domain decomposition and virtual control applied to the identification of a crack from boundary measurements and in section 5 , we present some numerical experiments which illustrate the efficiency of the method.

\section{Problem setting}

Let $\Omega$ be a bounded connected open domain of $\mathbb{R}^{2}$ with a smooth boundary $\Gamma_{c}$. We suppose that $\Omega$ contains exactly one crack $\sigma$ strictly included into $\Omega$ (see Fig. 1). In the whole paper, we assume that $\sigma$ is $C^{2}$ non self-intersecting compact curve with a finite length. We denote by $S_{1}$ and $S_{2}$ its extremities and by $\Omega_{\sigma}$ the domain $\Omega \backslash \sigma$.

Let $(f, g)$ be the measurement and the flux on the boundary $\Gamma_{c}=\partial \Omega$ which supposed to be accessible for measurement over a time interval $[0, T], T>0$. In the domain $\Omega_{\sigma}$, we define the following problem:

$$
\begin{cases}\partial_{t} u-\Delta u=0, & \text { in } \Omega_{\sigma} \times[0, T], \\ \nabla u \cdot v=g, & \text { on } \Gamma_{c} \times[0, T], \\ u=f, & \text { on } \Gamma_{c} \times[0, T], \\ \nabla u \cdot v=0, & \text { on } \sigma \times[0, T], \\ u(x, 0)=0, & \text { in } \Omega_{\sigma}\end{cases}
$$

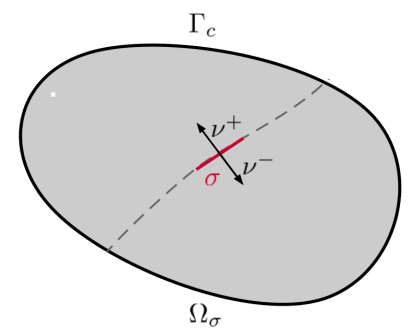

Fig. 1: Domain $\Omega_{\sigma}$.

\section{Identifiability for the inverse problem}

The inverse problem that we are discussing is the following: Setting a flux $g$ on the external part of the boundary $\Gamma_{c}$, and measuring $u$ on some non empty open subset $M$ of $\Gamma_{c}$, we try to recover the unknown crack $\sigma$.

We will give in the following theorem the identifiability result, which means that different pairs of crack with $C^{2}$-boundary may not produce same measurement on the boundary, provided the prescribed flux indeed generate singularity at both type of the actual crack. 

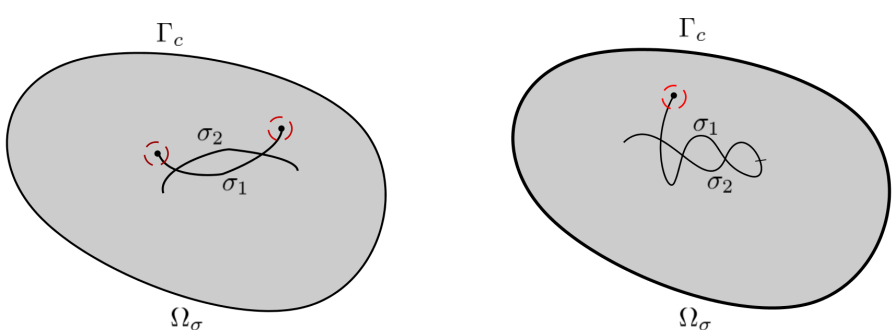

Fig. 2: Intersecting cracks with different cracktips.

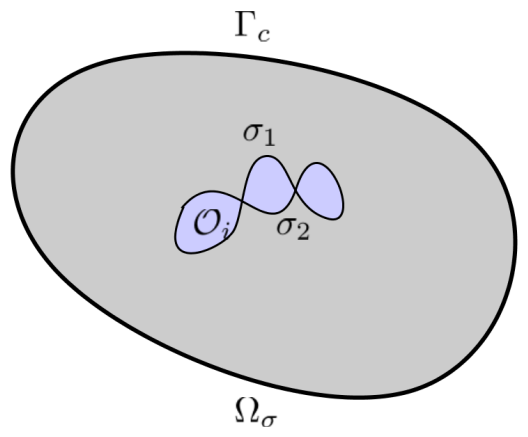

Fig. 3: Intersecting cracks with same cracktips.

Theorem 1. (Geometrical identifiability) Let $\sigma_{1}$ and $\sigma_{2}$ be two cracks with $C^{2}$-boundary. Assume a prescribed flux $g$ generating singularities at both extremities of the actual crack (for example $\sigma_{1}$ ), also generates equal measurements on $M$. Therefore $\sigma_{1} \equiv \sigma_{2}:=\sigma$, and $u_{1} \equiv u_{2}:=u$ on $\Omega_{\sigma} \times[0, T]$.

Proof. Let $u_{1}$, respectively, $u_{2}$ solve the forward problem in $\Omega_{\sigma_{1}} \times[0, T]$, respectively, in $\Omega_{\sigma_{2}} \times[0, T]$. Let $w:=u_{1}-u_{2}$ their difference, hence $w$ solve the following problem:

$$
\begin{cases}\partial_{t} w-\Delta w=0, & \text { in } \Omega \backslash\left(\sigma_{1} \cup \sigma_{2}\right) \times[0, T], \\ \nabla w \cdot v=0, & \text { on } \Gamma \times[0, T], \\ w=0, & \text { on } M \times[0, T], \\ w(x, 0)=0, & \text { on } \Omega .\end{cases}
$$

By unique continuation theorem, we can derive that $w \equiv 0$ on the external connected component $\Omega_{e}$ of $\Omega \backslash\left(\sigma_{1} \cup \sigma_{2}\right) \times$ $[0, T]$, i.e, the one having $M$ as part of its boundary. Assuming $\sigma_{1} \neq \sigma_{2}$, two situations may occur:

(a) The cracks are disconnected: in the case $\Omega_{e}=\Omega \backslash\left(\sigma_{1} \cup \sigma_{2}\right), u_{1}$ is not continuous across $\sigma_{1}$ because of its singular parts but $u_{2}$ is continuous across $\sigma_{1}$. Then, this situation is not possible.

(b) The cracks are intersecting: in that case the cracks cannot have different endpoints (see Fig. 2), because $u_{1}$ have singular parts. Otherwise, $u_{2}$ would be singular at the vicinity of an endpoint of $\sigma_{1}$ which is an internal point either to $\Omega \backslash \sigma_{2}$. Since, $\sigma_{1}$ and $\sigma_{2}$ have the same crack tips (see Fig. 3), then the boundary of $\Omega_{e}$ is composed by $\Gamma$ and upper or lower parts of $\sigma_{1}$ and $\sigma_{2}$. Let $\mathscr{O}_{i}$ be any connected component of $\Omega \backslash\left(\sigma_{1} \cup \sigma_{2} \cup \Omega_{e}\right)$. This boundary is necessarily composed by part of $\sigma_{1}$ and $\sigma_{2}$.

The functions $u_{1}, \nabla u_{1} \cdot v$ and $\nabla u_{2} \cdot v$ are continuous across $\sigma_{2}$. On the other hand $u_{1} \equiv u_{2}$ in $\Omega_{e} \times[0, T]$. Hence, 
$\nabla u_{1} \cdot v=\nabla u_{2} \cdot v$ on $\partial \Omega_{e} \times[0, T]$, including the external part of $\sigma_{1}$ and $\sigma_{2}$ then we have

$$
\nabla w \cdot v=0 \text { on } \sigma_{2} \times[0, T] .
$$

Following the same procedure, on can derive that

$$
\nabla w \cdot v=0 \text { on } \sigma_{1} \times[0, T]
$$

where $w$ is solution of the homogeneous heat equation with homogeneous Neumann data on $\sigma_{1}$. By Cauchy-Kovalevskaya theorem [22], one can deduce that $[w]$ is real analytic on $\sigma_{1}$ then $w=k_{i}$ on $\sigma_{1} \cap \partial \mathscr{O}_{i} \times[0, T]$ and since $w$ is null in $\Omega_{e}$, this yields: where

$$
\left[u_{1}\right]=[w]= \pm k_{i}, \text { on } \sigma_{1} \cap \overline{\mathscr{O}_{i}} \times[0, T]
$$

where $\left[u_{1}\right]$ is piecewise constant on $\sigma_{1}$ which is not possible unless it is constant on the whole of $\sigma_{1}$ since no discontinuity is allowed to function in $H_{00}^{\frac{1}{2}}\left(\sigma_{1} \times[0, T]\right)$. Hence:

$$
\left[u_{1}\right]=[w]=k_{i} \text {, on } \sigma_{1} \times[0, T],
$$

where $\left[u_{1}\right] \in H_{00}^{\frac{1}{2}}\left(\sigma_{1} \times[0, T]\right)$ and thus vanishes at the endpoints of $\sigma_{1}$. The constant $k$ cannot be other then zero, making $u_{1}$ continues across $\sigma_{1} \times[0, T]$ and hence not singular, which contradicts the assumptions made on the flux, the cracks cannot these intersect either. This leads to $\sigma:=\sigma_{1} \equiv \sigma_{2}$ an according to $u:=u_{1} \equiv u_{2}$ on $\Omega_{\sigma} \times[0, T]$.

Remark 1 The solution $u$ of the heat equation admits the following decomposition into a regular part and a singular one:

$$
u(x, t)=u_{\text {reg }}(x, t)+u_{\text {sing }}(x, t) \text { in } \mathscr{V} \backslash \sigma \times[0, T],
$$

where:

$-\mathscr{V}$ is a neighborhood of $\sigma$.

$-u_{\text {reg }} \in H^{2}\left(\Omega_{\sigma} \times[0, T]\right)$.

$-u_{\text {sing }}$ is written in front of the crack by: $u_{\text {sing }}=\sum_{i=1,2} c_{i}(t) r_{i}^{\frac{1}{2}} \sin \left(\frac{\theta_{i}}{2}\right)$.

$-\left(r_{i}, \theta_{i}\right)$ are polar coordinates centered at $S_{i}$ such that the half-lines $\theta_{i}=0$ and $\theta_{i}=2 \pi$ are tangent to $\sigma$ at $S_{i}$.

$-c_{i}(t)$ is the coefficient of singularity related to the extremity $S_{i}$.

Remark 2 In [17], Grisvard gave a characterization convolution of the coefficients of singularities by using the Laplace equation and the Fourier transform. Moussaoui [26], also, obtained an explicit expansion based on Bessel functions.

\section{Domain decomposition and virtual control}

\subsection{Statement of the inverse problem}

We assume that we know the interface plane $\Gamma_{i}$ containing the crack $\sigma$. Then, the inverse problem consists in finding the position of the crack on $\Gamma_{i}$, while, having $u$ which solves the following equation:

$$
\begin{cases}\partial_{t} u-\Delta u=0 & \text { in } \Omega_{\sigma} \times[0, T], \\ \nabla u \cdot v=g & \text { on } \Gamma_{c} \times[0, T], \\ u=f & \text { on } \Gamma_{c} \times[0, T], \\ u(x, 0)=0 & \text { in } \Omega,\end{cases}
$$




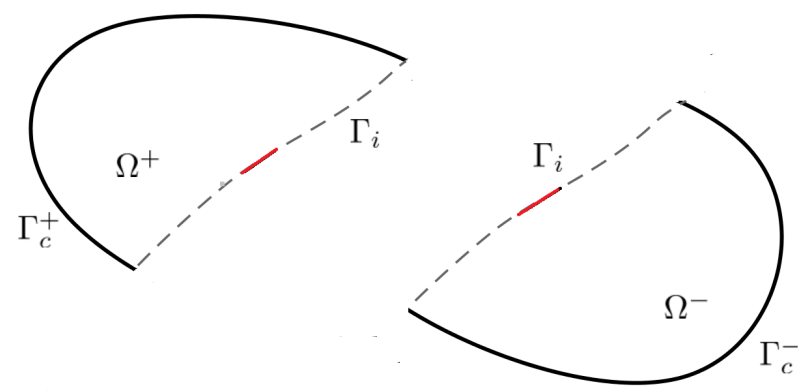

Fig. 4: Decomposition of the domain $\Omega$ into two adjacent sub-domains.

where $(f, g)$ is a pair related to measurement and flux on $\Gamma \times[0, T]$ of the direct problem (1). We suppose that the domain $\Omega$ is partitioned into two adjacent sub-domains $\Omega^{+}$and $\Omega^{-}$such that $\Omega=\Omega^{+} \cup \Omega^{-}$, and $\Gamma_{i}=\overline{\Omega^{+}} \cap \overline{\Omega^{-}}$is the common interface between them (see Fig. 4). Let us define the following Cauchy problem in the sub-domain $\Omega^{+}$:

$$
\begin{cases}\partial_{t} u^{+}-\Delta u^{+}=0 & \text { in } \Omega^{+} \times[0, T] \\ \nabla u^{+} \cdot v=g & \text { on } \Gamma_{c}^{+} \times[0, T], \\ u^{+}=f, & \text { on } \Gamma_{c}^{+} \times[0, T], \\ u^{+}(x, 0)=0 & \text { in } \Omega^{+}\end{cases}
$$

With $(f, g)$ defined in (1) as Dirichlet and Neumann boundary conditions in $\Gamma_{c}^{+}$.

Similarly, let $(f, g)$ be a data on $\Gamma_{c}^{-}$we define in the sub-domain $\Omega^{-}$the following Cauchy problem:

$$
\begin{cases}\partial_{t} u^{-}-\Delta u^{-}=0 & \text { in } \Omega^{-} \times[0, T], \\ \nabla u^{-} \cdot v=g & \text { on } \Gamma_{c}^{-} \times[0, T], \\ u^{-}=f & \text { on } \Gamma_{c}^{-} \times[0, T], \\ u^{-}(x, 0)=0 & \text { in } \Omega^{-} .\end{cases}
$$

The Cauchy problems $(\mathrm{P}+)$ and $(\mathrm{P}-)$ are ill-posed in the sense of Hadamard [18]. Existence of solutions for arbitrary Cauchy data $f$ and $g$ is not guaranteed and depends on the compatibility of $f$ and $g$. The theoretical results of existence and uniqueness of the problems $(\mathrm{P}+)$ and $(\mathrm{P}-)$ are given in [21].

The localization of the crack can be achieved by solving Cauchy porblems $(\mathrm{P}+)$ and $(\mathrm{P}-)$. In fact, it is easy to see that when we solve both problems $(\mathrm{P}+)$ and $(\mathrm{P}-)$ it amounts to solve the Cauchy problem $(\mathrm{P})$. In addition, the crack can be appear as parts of the boundary $\Gamma_{i}$ where the jump $[[u]]=u^{+}-u^{-}$is not null.

\subsection{Time discretization of the Cauchy problems}

From the numerical resolution of the equation $(\mathrm{P}+)$ and $(\mathrm{P}-)$ we consider a time-discretization based on forward Euler finite difference scheme. First, let us introduce the discretization of the time interval $[0, T]$ into $n$ sub-intervals by:

$$
0=t^{0}<t^{1}<\cdots<t^{n-1}<t^{n}=T, n \in \mathbb{N}^{*}
$$


where $t^{n}=n \delta t$, and, $\delta t=\frac{T}{n}=t^{n}-t^{n-1}$ is the time step. We approximate $\partial_{t} u$ by the forward Euler scheme as follows:

$$
\partial_{t} u=\frac{u^{n+1}-u^{n}}{\delta t}, \text { at } t=t^{n+1}
$$

Therefore, we look for $u^{+^{n+1}}$ and $u^{-^{n+1}}$ which are solutions of the following problems:

$$
\begin{aligned}
& \begin{cases}u^{+^{n+1}}-\delta t \Delta u^{+^{n+1}}=u^{+^{n}} & \text { in } \Omega^{+}, \\
\nabla u^{+^{n+1}} \cdot v=g & \text { on } \Gamma_{c}^{+}, \\
u^{+^{n+1}}=f & \text { on } \Gamma_{c}^{+} .\end{cases} \\
& \begin{cases}u^{-^{n+1}}-\delta t \Delta u^{-^{n+1}}=u^{-^{n}} & \text { in } \Omega^{-}, \\
\nabla u^{-n+1} \cdot v=g & \text { on } \Gamma_{c}^{-}, \\
u^{-^{n+1}}=f & \text { on } \Gamma_{c}^{-} .\end{cases}
\end{aligned}
$$

The goal is to find, at each step of time, the solution ${u^{+}}^{n+1}$ and $u^{-n+1}$ in $\Omega^{+}$and $\Omega^{-}$, respectively.

\subsection{Virtual control approach}

In the sequel, we explain the domain decomposition and the virtual control approach applied for the problem (2), and the application of this approach to problem (3) is similar. For brevity's sake, we set $\lambda:=\lambda^{n}\left(\lambda\right.$ at $\left.t^{n}\right)$ and we use the notation $u$ instead of $u^{+}$in the problem (2).

We search $u_{D}^{n+1}$ and $u_{N}^{n+1}$ solutions of the following well posed problems:

$$
\begin{aligned}
& \begin{cases}u_{D}^{n+1}-\delta t \Delta u_{D}^{n+1}=u^{n} & \text { in } \Omega^{+}, \\
u_{D}^{n+1}=f & \text { on } \Gamma_{c}^{+}, \\
u_{D}^{n+1}=\lambda & \text { on } \Gamma_{i} .\end{cases} \\
& \begin{cases}u_{N}^{n+1}-\delta t \Delta u_{N}^{n+1}=u^{n} & \text { in } \Omega^{+}, \\
\nabla u_{N}^{n+1} \cdot v=g & \text { on } \Gamma_{c}^{+}, \\
u_{N}^{n+1}=\lambda & \text { on } \Gamma_{i} .\end{cases}
\end{aligned}
$$

The function $\lambda \in H^{\frac{1}{2}}\left(\Gamma_{i}\right)$, which represents the virtual control, is chosen such that $u_{D}$ and $u_{N}$ "adjust" in the best possible way in $\Omega^{+}$.

To this aim, we introduce the following minimization problem:

$$
\inf _{\lambda \in H^{\frac{1}{2}}\left(\Gamma_{i}\right)} J(\lambda)
$$

where the cost functional $J($.$) is given by:$

$$
J(\cdot)=\frac{\delta t}{2} \int_{\Omega^{+}}\left(\nabla u_{D}^{n+1}(\cdot)-\nabla u_{N}^{n+1}(\cdot)\right)^{2} d x+\frac{1}{2} \int_{\Omega^{+}}\left(u_{D}^{n+1}(\cdot)-u_{N}^{n+1}(\cdot)\right)^{2}
$$


The minimization problem (6) has a unique solution. Now, for simplicity, we use the notation $u_{N}^{n+1}$ by $u_{N}$ instead of $u_{D}^{n+1}$ by $u_{D}$, respectively. Indeed, let us rewrite the solution $u_{D}$ and $u_{N}$ respectively of (4) and (5) as:

$$
u_{D}=u_{D}^{0}+u_{D}^{\lambda} ; \quad u_{N}=u_{N}^{0}+u_{N}^{\lambda},
$$

where $u_{D}^{0}$ and $u_{N}^{0}$ depend only on the data $\mathrm{f}$ and $\mathrm{g}, u_{D}^{\lambda}$ and $u_{N}^{\lambda}$ depend only on $\lambda$, and satisfy

$$
\begin{gathered}
u_{D}^{0}-\delta t \Delta u_{D}^{0}=v_{D} \text { in } \Omega^{+}, u_{D}^{0}=f \text { on } \Gamma_{c}^{+}, u_{D}^{0}=0 \text { on } \Gamma_{i}, \\
u_{D}^{\lambda}-\delta t \Delta u_{D}^{\lambda}=0 \text { in } \Omega^{+}, u_{D}^{\lambda}=0 \text { on } \Gamma_{c}^{+}, u_{D}^{\lambda}=\lambda \text { on } \Gamma_{i},
\end{gathered}
$$

and

$$
\begin{gathered}
u_{N}^{0}-\delta t \Delta u_{N}^{0}=v_{N} \text { in } \Omega^{+}, \nabla u_{N}^{0} \cdot v=g \text { on } \Gamma_{c}^{+}, u_{N}^{0}=0 \text { on } \Gamma_{i}, \\
u_{N}^{\lambda}-\delta t \Delta u_{N}^{\lambda}=0 \text { in } \Omega^{+}, \nabla u_{N}^{\lambda} \cdot v=0 \text { on } \Gamma_{c}^{+}, u_{N}^{\lambda}=\lambda \text { on } \Gamma_{i},
\end{gathered}
$$

where $v_{D}=u_{D}^{n}$ and $v_{N}=u_{N}^{n}$. Then

$$
J(\lambda)=\frac{1}{2} Q(\lambda)+\mathscr{L}(\lambda)
$$

where the quadratic functional $Q$ is given by

$$
Q(\lambda)=\int_{\Omega^{+}}\left(\nabla u_{D}^{\lambda}-\nabla u_{N}^{\lambda}\right)^{2}+\frac{1}{\delta t} \int_{\Omega^{+}}\left(u_{D}^{\lambda}-u_{N}^{\lambda}\right)^{2},
$$

and $\mathscr{L}$ is an affine functional. Consequently, if the function $\lambda$ is smooth enough, one can define the following semi-norm on the space of admissible functional $\lambda$

$$
\||\lambda|\|:=(Q(\lambda))^{\frac{1}{2}} .
$$

Therefore, the minimization of $J($.$) admits a solution in the space of admissible functional of \lambda$ obtained by completion for the semi-norm (11).

Actually, this is a norm. Indeed if $Q(\lambda)=0$, then $u_{D}^{\lambda}=u_{N}^{\lambda}=u^{\lambda}$ in $\Omega^{+}$. From (9b) we have $u^{\lambda}-\delta t \Delta u^{\lambda}=0$ in $\Omega^{+}$, and $u^{\lambda}=0$ in $\Gamma^{+}$. Moreover, from (10b) we obtain $\nabla u^{\lambda} \cdot v=0$ on $\Gamma^{+}$. Thus by the continuation theorem it follows that $u^{\lambda}=0$ in $\Omega^{+}$. Consequently, $\lambda=0$ which leads to the conclusion that (11) is a norm.

Remark. The inverse problem $(\mathrm{P}+)$ is formulated into a minimization problem:

$$
\left\{\begin{array}{l}
\text { Find } \lambda \in H^{\frac{1}{2}}\left(\Gamma_{i}\right) \text { such that, } \\
\tilde{J}(\lambda)=\inf _{\lambda \in H^{\frac{1}{2}}\left(\Gamma_{i}\right)} J(\lambda)
\end{array}\right.
$$

where $J($.$) is the energy-type error functional. This functional has been already introduced for data completion in the$ framework of Laplace equation [3,5].

The approach that we follow consists in solving an interfacial equation arising from the optimality conditions of (12) instead of finding the minimizer of $J$ using classical descent gradient algorithms. In fact, we have the following result

Theorem 2. Let $(f, g)$ be a compatible data, then the minimum of $J$ is reached when

$$
\begin{aligned}
u_{D} & =u_{N}+c \text { on } \Gamma_{i}, \\
\nabla u_{D} \cdot v & =\nabla u_{N} \cdot v \text { on } \Gamma_{i},
\end{aligned}
$$


Above, $c$ is a constant.

Proof. The first condition is follows directly by choosing $\lambda=u_{D}=u_{N}$. For the second one, we compute the first derivative of $J$ over $\lambda\left(=u_{D}\right.$ or $u_{N}$ ), using the boundary conditions of equation (4) and (5), we have

$$
\begin{aligned}
\nabla J . \lambda= & \int_{\Omega^{+}}\left(\nabla u_{D}^{\lambda}-\nabla u_{N}^{\lambda}\right)\left(\nabla u_{D}-\nabla u_{N}\right)+\frac{1}{\delta t} \int_{\Omega^{+}}\left(u_{D}^{\lambda}-u_{N}^{\lambda}\right)\left(u_{D}-u_{N}\right) \\
= & \int_{\Omega^{+}}\left(\nabla u_{D}-\nabla u_{N}\right) \cdot \nabla u_{D}^{\lambda}-\left(\nabla u_{D}-\nabla u_{N}\right) \cdot \nabla u_{N}^{\lambda} \\
& +\frac{1}{\delta t} \int_{\Omega^{+}}\left(u_{D}-u_{N}\right) u_{D}^{\lambda}-\left(u_{D}-u_{N}\right) u_{N}^{\lambda} \\
= & -\int_{\Omega^{+}} \Delta\left(u_{D}-u_{N}\right) u_{D}^{\lambda}+\int_{\partial \Omega^{+}} u_{D}^{\lambda} \nabla\left(u_{D}-u_{N}\right) \cdot v \\
& +\int_{\Omega^{+}} \Delta u_{N}^{\lambda}\left(u_{D}-u_{N}\right)-\int_{\partial \Omega^{+}}\left(u_{D}-u_{N}\right) \nabla u_{N}^{\lambda} \cdot v \\
& +\frac{1}{\delta t} \int_{\Omega^{+}}\left(u_{D}-u_{N}\right) u_{D}^{\lambda}-\left(u_{D}-u_{N}\right) u_{N}^{\lambda}
\end{aligned}
$$

Since

$$
\nabla u_{N}^{\lambda} \cdot v=0 \text { on } \Gamma_{c}^{+}, u_{D}-u_{N}=0 \text { on } \Gamma_{i} \text { and } u_{D}^{\lambda}=0 \text { on } \Gamma_{c}^{+}
$$

We obtain:

$$
\nabla J \cdot \lambda=\int_{\Gamma_{i}} u_{D}^{\lambda} \nabla\left(u_{D}-u_{N}\right) \cdot v=\int_{\Gamma_{i}} \lambda \nabla\left(u_{D}-u_{N}\right) \cdot v .
$$

From the first order optimal condition, we obtain $\nabla J \cdot \lambda=0$. Then, we have:

$$
\nabla u_{D} \cdot v-\nabla u_{N} \cdot v=0 \text { on } \Gamma_{i}
$$

Remark 3 By a decomposition (8), the equation (13) is equivalent to

$$
\nabla\left(u_{D}^{0}-u_{N}^{0}\right) \cdot v=-\nabla\left(u_{D}^{\lambda}-u_{N}^{\lambda}\right) \cdot v \text { on } \Gamma_{i}
$$

\subsection{Stecklov-Poincaré operator}

To solve the interface equations (4) and (5), we follow the techniques of domain decomposition using the the StecklovPoincaré operator. Then, the interface conditions (14) can be equivalently expressed as the following linear system

$$
S \lambda=\chi
$$

where

$$
\chi=-\nabla\left(u_{D}^{0}-u_{N}^{0}\right) \cdot v
$$

and

$$
S \lambda=S_{D} \lambda-S_{N} \lambda=\nabla u_{D}^{\lambda} \cdot v-\nabla u_{N}^{\lambda} \cdot v
$$

where

$$
\begin{aligned}
S_{D}: H^{\frac{1}{2}}\left(\Gamma_{i}\right) & \rightarrow H^{-\frac{1}{2}}\left(\Gamma_{i}\right) \\
\lambda & \mapsto \nabla u_{D}^{\lambda} \cdot v, \\
S_{N}: H^{\frac{1}{2}}\left(\Gamma_{i}\right) & \rightarrow H^{-\frac{1}{2}}\left(\Gamma_{i}\right) \\
\lambda & \mapsto \nabla u_{N}^{\lambda} \cdot v .
\end{aligned}
$$


$S_{D}$ and $S_{N}$ are so-called Stecklov-Poincaré operators. These operators are widely used in the domain decomposition community (see $[6,10,27])$. There are several ways to solve this linear system of equations. Here, we use the following iterative preconditioned gradient algorithm:

$$
\left\{\begin{array}{l}
\lambda_{0} \text { is given, } \\
\lambda_{k+1}=\lambda_{k}+\rho\left(S_{D}^{-1}\left(S \lambda_{k}-\chi\right), k \geq 0\right.
\end{array}\right.
$$

where $\rho$ is a coefficient of relaxation and $S_{D}$ is the preconditioning operator. Thus, each iteration requires to compute $S \lambda_{k}$ by solving problems (4), (5) and the system $S \mu=S \lambda_{k}$. This is achieved by solving the following problem:

$$
\begin{cases}w^{n+1}-\delta t \Delta w^{n+1}=w^{n} & \text { in } \Omega^{+}, \\ w^{n+1}=0 & \text { on } \Gamma_{c}^{+}, \\ w^{n+1}=S \lambda_{k} & \text { on } \Gamma_{i},\end{cases}
$$

where $w^{n}$ is the solution of the problem (15) in the previous step. We initialize by $w^{0}=0$. Then $\mu=w^{n+1}$ on $\Gamma_{i}$ at time $t^{n+1}$.

These three problems (4), (5) and (15) must be discretized in space for numerical simulations by a finite element method.

\section{Numerical results}

In this work, all the PDEs are solved with the finite-element open source software FreeFem++ [20].

\subsection{Single crack identification}

We consider a computational domain $\Omega$ which is a $2 D$ annular section with radius $r_{1}=1$ and $r_{2}=3$. Let $\Gamma_{c}^{+}, \Gamma_{c}^{-}$and $\Gamma_{i}$ be the outer circle, the inner one, and the circle of radius $r_{3}=2$, respectively ( see Fig. 5). The crack in this case is given in polar coordinate by

$$
\sigma_{1}=\left\{r=2, \frac{\pi}{3} \leq \theta \leq \frac{13 \pi}{30}\right\} .
$$

We note by $\Omega^{+}$the sub-domain limited by $\Gamma_{c}^{+}$and $\Gamma_{i}, \Omega^{-}$the domain with boundary $\Gamma_{c}^{-}$and $\Gamma_{i}$. We consider the mesh where we have set: 300 nodes on $\Gamma_{c}^{+}, 100$ nodes on $\Gamma_{c}^{-}, 210$ on $\Gamma_{i} \backslash \sigma_{1}$ and 20 nodes on $\sigma_{1}$. For the discretization of the time derivative, we consider a final time $T=10$ and a time step $\delta t=1$. The stopping criteria in each problem is $J(\lambda) \leq \xi$, where $\xi$ is a given tolerance level and $J$ the energy-type error functional defined in (7). In this test, we take the case of $\xi=10^{-2}$. A synthetic data are generated by solving the two following forward problems

$$
\begin{aligned}
& \begin{cases}\partial_{t} u^{+}-\Delta u^{+}=0 & \text { in } \Omega^{+} \times[0, T], \\
\nabla u^{+} \cdot v=G & \text { on } \Gamma_{c}^{+} \times[0, T], \\
u^{+}=F & \text { on } \Gamma_{c}^{+} \times[0, T], \\
u^{+}(x, 0)=0 & \text { in } \Omega^{+} .\end{cases} \\
& \begin{cases}\partial_{t} u^{-}-\Delta u^{-}=0 & \text { in } \Omega^{-} \times[0, T], \\
\nabla u^{-} \cdot v=G & \text { on } \Gamma_{c}^{-} \times[0, T], \\
u^{-}=F & \text { on } \Gamma_{c}^{-} \times[0, T], \\
u^{-}(x, 0)=0 & \text { in } \Omega^{-},\end{cases}
\end{aligned}
$$




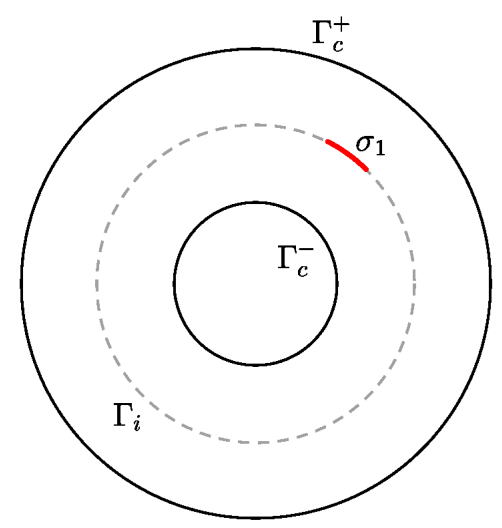

$\Omega$

Fig. 5: Computational domain $\Omega$ with single crack.

where $F=x+y$ and $G=t$.

In Fig.6, we display the exact Dirichlet data $\left.u_{\text {exact }}\right|_{\Gamma_{i}}$ and Dirichlet data $\left.u^{+}\right|_{\Gamma_{i}}$ and $\left.u^{-}\right|_{\Gamma_{i}}$ at different time steps, i.e., $t=2,4,6$ and 8, respectively. We notice that we have a peak in the curves which corresponds the crack position. Over time iterations, we can see the efficiency of the proposed method and the crack position is progressively better approximated. Indeed, the height of the peak is being more important which give more accuracy in the identification of the crack position. In Fig. 7, we display the difference between the two solutions $u^{+}$and $u^{-}$on $\Gamma_{i}$ at $t=2,4,6,8$. As expected, the crack is located where the jump between the two solutions is very important.

\subsection{Multiple crack zone identification}

\subsubsection{Cracks with same size}

In this case, we consider the same computational domain as in the previous example and we consider two cracks with same size $l\left(l=\frac{\pi}{10} \simeq 0.31\right.$ ) (see Fig.8). The crack $\sigma_{1}$, which is defined in (16) and a second crack $\sigma_{2}$ which is given in polar coordinate by:

$$
\sigma_{2}=\left\{r=2, \frac{3 \pi}{2} \leq \theta \leq \frac{8 \pi}{5}\right\} .
$$

In Fig. 9, we present numerical result for the case when the two cracks have same sizes for different time. We present the comparison between the exact and the reconstructed solution on $\Gamma_{i}$ for the Dirichlet data for the two Cauchy problems (P1) and (P2). We believe that there is merit in the proposed procedure. In Fig. 10, we display the difference between the solutions $u^{+}$and $u^{-}$on $\Gamma_{i}$ at different time steps, i.e, $t=2,4,6,8$. We obtain two peacks at two different positions which correspond to the position of cracks.

\subsection{2 cracks of different size}

Now, we consider the same computational domain as in the previous example and we consider two cracks $\sigma_{3}$ and $\sigma_{4}$ with different size respectively $l_{3}=d$ and $l_{4}=2 d$ with $d=0.2$. 
a)

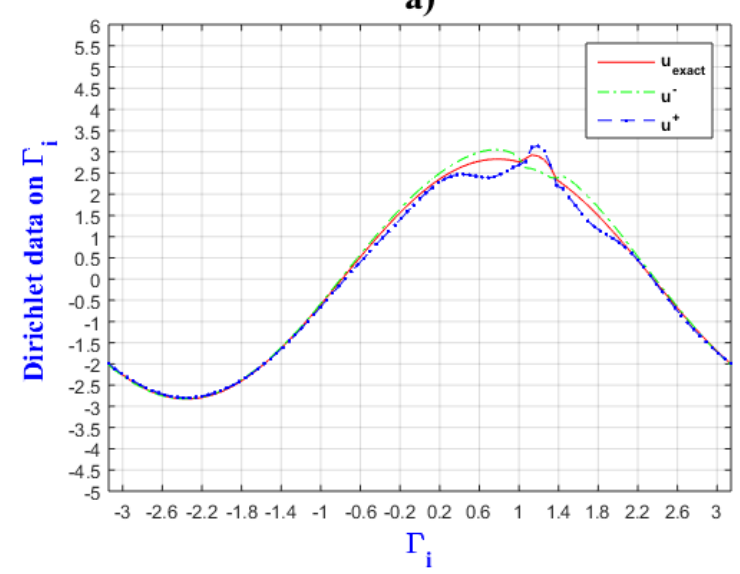

c)

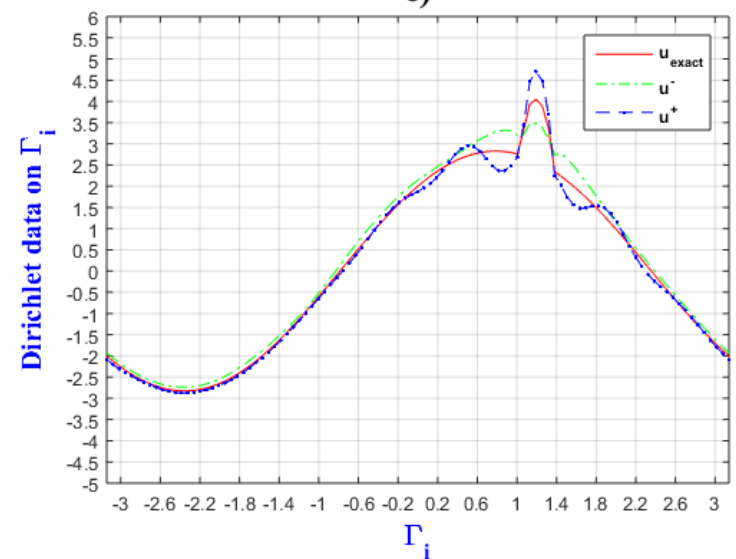

b)

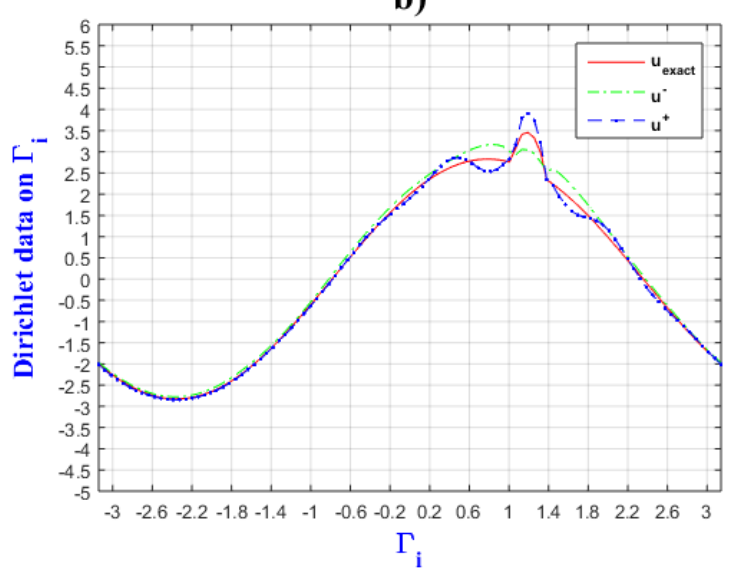

d)

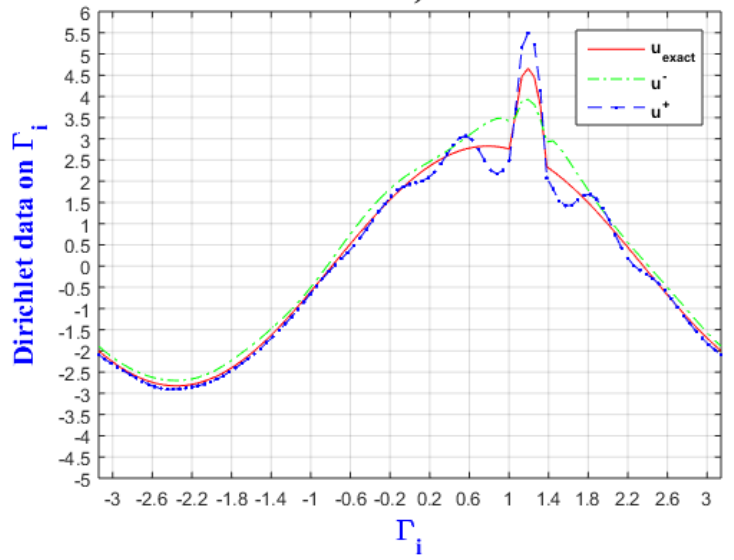

Fig. 6: Single crack: Reconstruction of the Dirichlet date $u^{+}, u^{-}$and $u_{\text {exact }}$ on $\Gamma_{i}$ for a test case with a) $\left.\left.\mathrm{T}=2 \mathrm{~b}\right) \mathrm{T}=4 \mathrm{c}\right) \mathrm{T}=6$ d) $\mathrm{T}=8, \xi=10^{-2}, \delta t=1$.

The cracks $\sigma_{3}$ and $\sigma_{4}$ is defined respectively in polar coordinate by :

$$
\begin{gathered}
\sigma_{3}=\left\{r=2, \frac{\pi}{4} \leq \theta \leq \frac{\pi}{4}+d\right\}, \\
\sigma_{4}=\left\{r=2, \frac{3 \pi}{2}-2 d \leq \theta \leq \frac{3 \pi}{2}\right\} .
\end{gathered}
$$

In Figs. 11 and 12, the reconstruction are given on $\Gamma_{i}$ for different time step $t=2,4,6,8$ for the case when the two cracks are different sizes. Having cracks zones of different sizes does not change the results.

\subsection{Variation of the parameters}

In the general theory of regularization of inverse problems, it is preferable to adjust the different parameters (time step, regularization parameter, mesh size, stopping criterion) in the model in order to achieve a numerical approximation of the solution of the inverse problem with high accuracy. In addition, the numerical solution may be very sensitive with respect to one or more of these parameters. Then, there is a careful interplay between the various parameters and so it's necessary to change one of them by keeping the others fixed to thereby see its influence on the obtained numerical reconstruction. 
a)

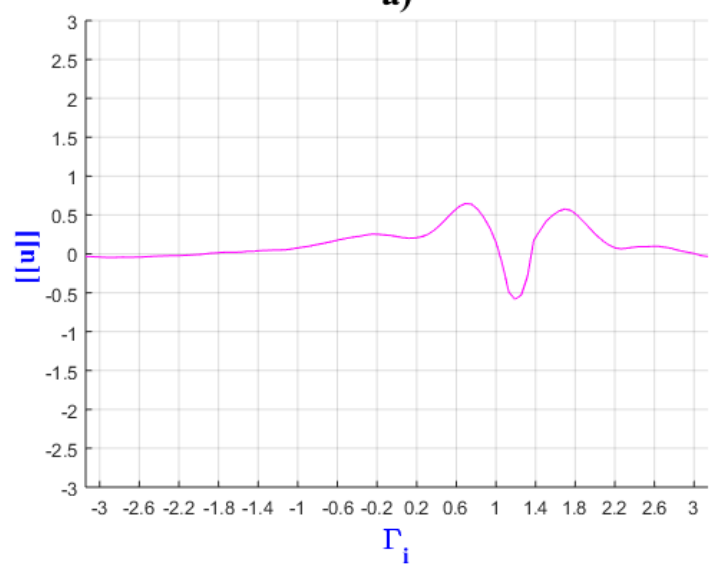

c)

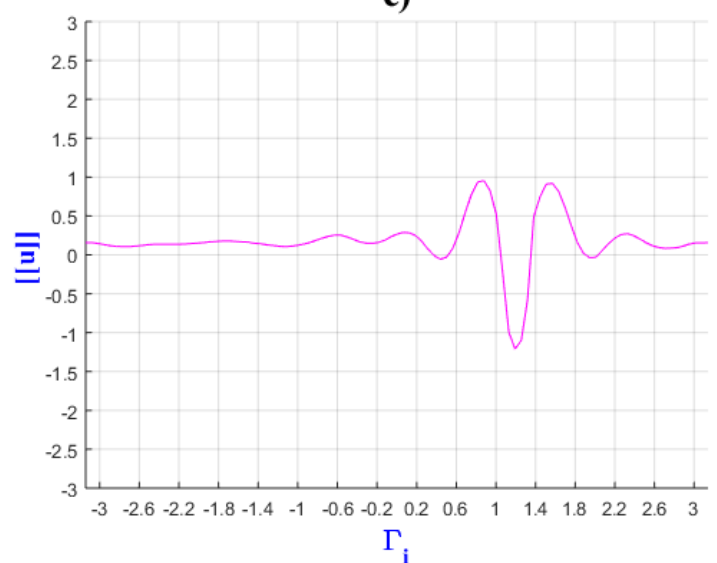

b)

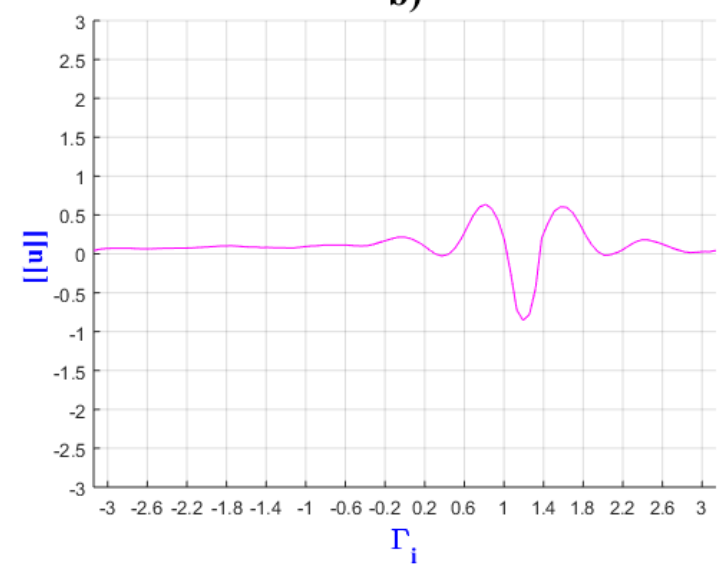

d)

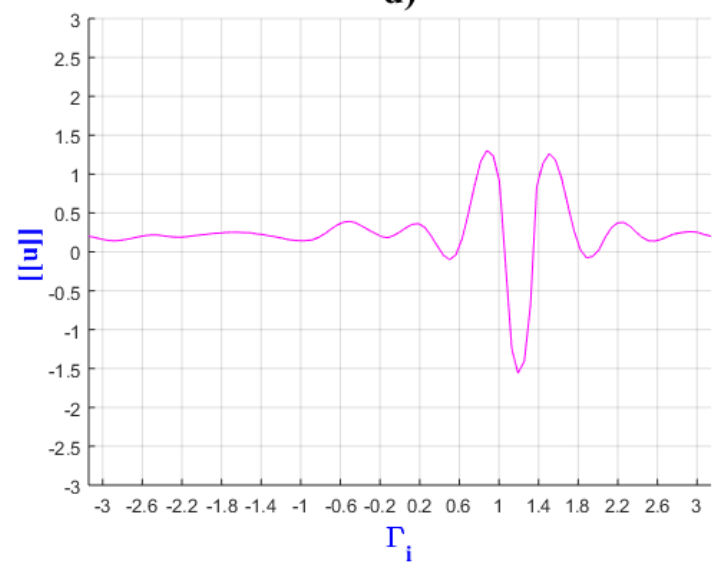

Fig. 7: Single crack: Reconstructed jump $[[u]]=u^{+}-u^{-}$on $\Gamma_{i}$ for a test case with $\mathrm{t}=2$ a), b) $\mathrm{t}=4$, c) $\mathrm{t}=6$ and $\left.\mathrm{d}\right) \mathrm{t}=8$, $\xi=10^{-2}, \delta t=1$.

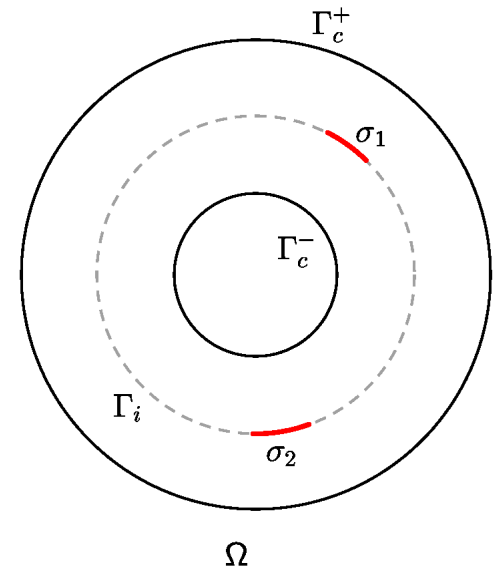

Fig. 8: Computational domain $\Omega$ with multiple cracks . 
a)

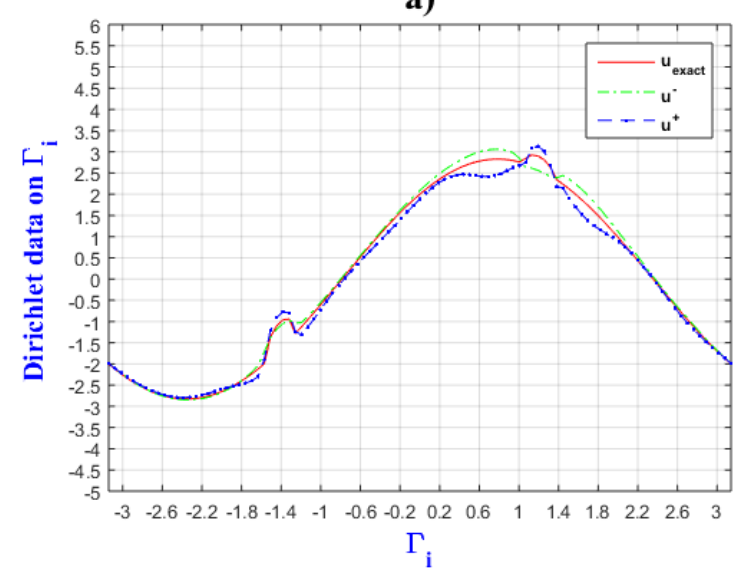

c)

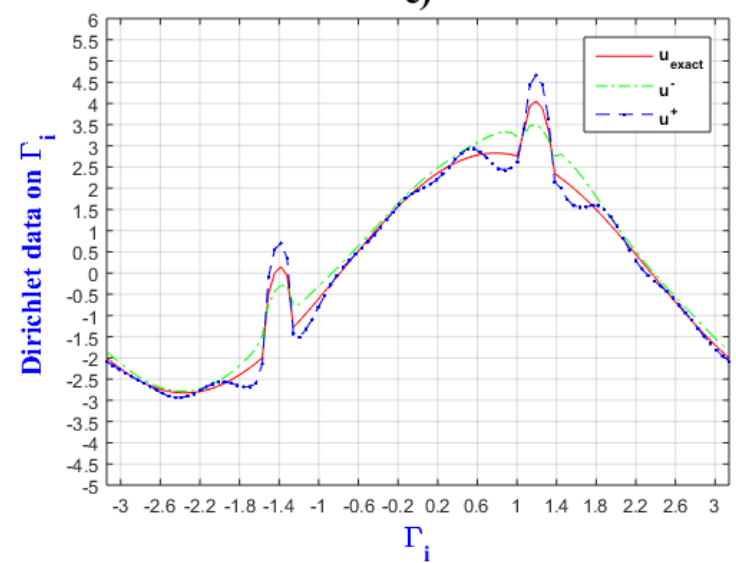

b)

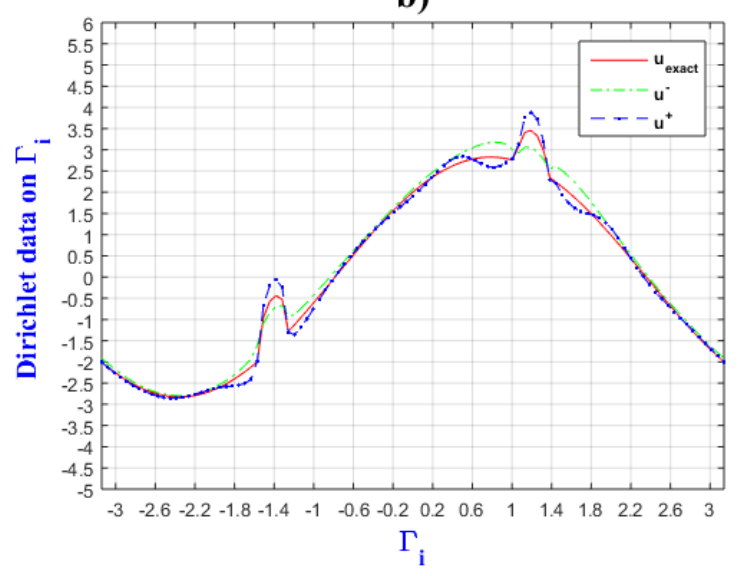

d)

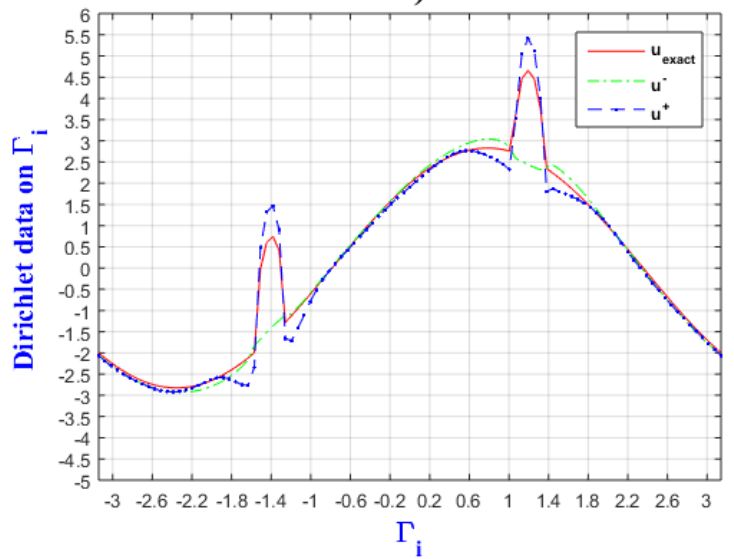

Fig. 9: Cracks with same size: Reconstruction of the Dirichlet date $u^{+}, u^{-}$and $u_{\text {exact }}$ on $\Gamma_{i}$ for a test case with a) $\mathrm{t}=2 \mathrm{~b}$ ) $\mathrm{t}=4$ c) $\mathrm{t}=6 \mathrm{~d}) \mathrm{t}=8, \xi=10^{-2}, \delta t=1$.

\subsubsection{Changing the mesh size}

In this step, we return for the test of single crack and we fix the following parameters : $\delta t=1$, tolerance $\xi=10^{-2}$, and $t=2$ and we address the stability of the jump with respect to the mesh size. To begin with, changing the mesh size influences the number of iterations needed before reaching the chosen stopping criteria. The dependence of the number of iterations on the mesh size are given in table 1 from which it can be seen that increasing the mesh size decreases the number of iterations, slightly. More mesh points make the discretized problem to be closer to the original Cauchy problem, and in particular more numerically unstable, forcing an earlier termination of the iterations. The accuracy of the reconstructions are somewhat improved as illustrated in Fig. 13. In particular, the reconstruction of the jump goes up several factors when increasing the mesh size. This is some evidence in the previous examples that the numerical results can be improved by elaborating on the parameters.

\subsubsection{Variation of the regularization parameter $\gamma$}

We consider now, in the case of single crack, the mesh size fixed and composed of 100 nodes on $\Gamma_{c}^{-}, 300$ on nodes $\Gamma_{c}^{+}$ and 230 nodes on $\Gamma_{i}$. We present a problem (P1) and (P2) with different regularizing parameter $\gamma$. We note $\gamma_{1}$ for problem 
a)

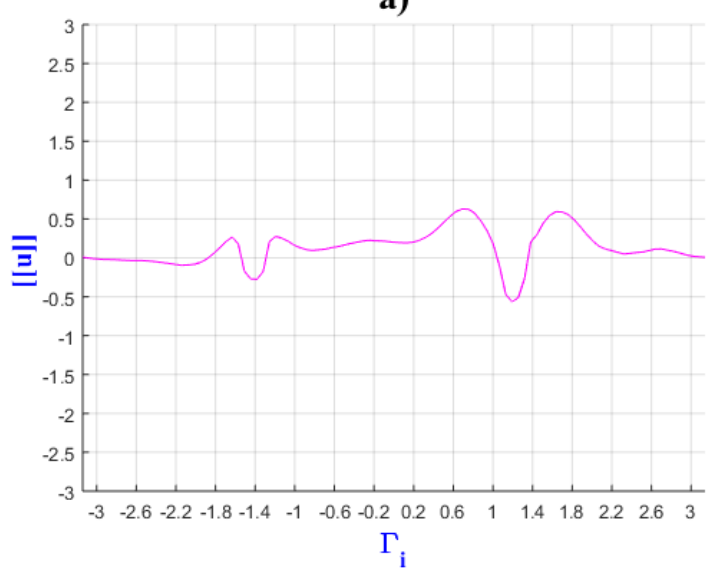

c)

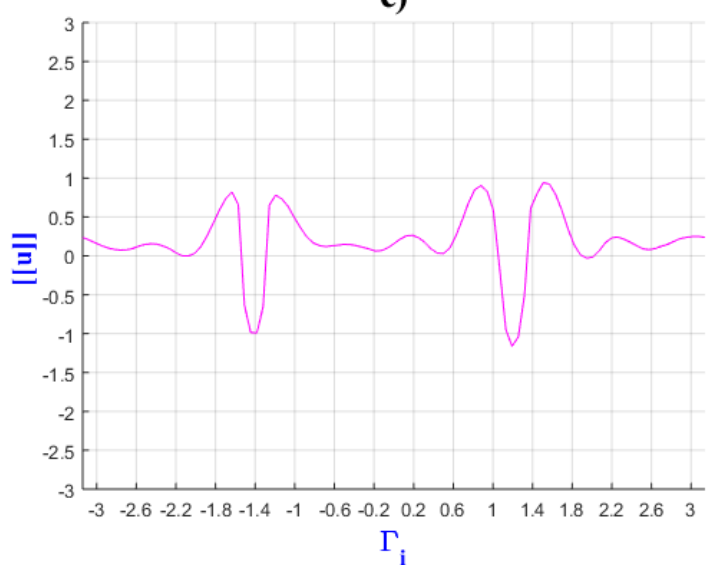

b)

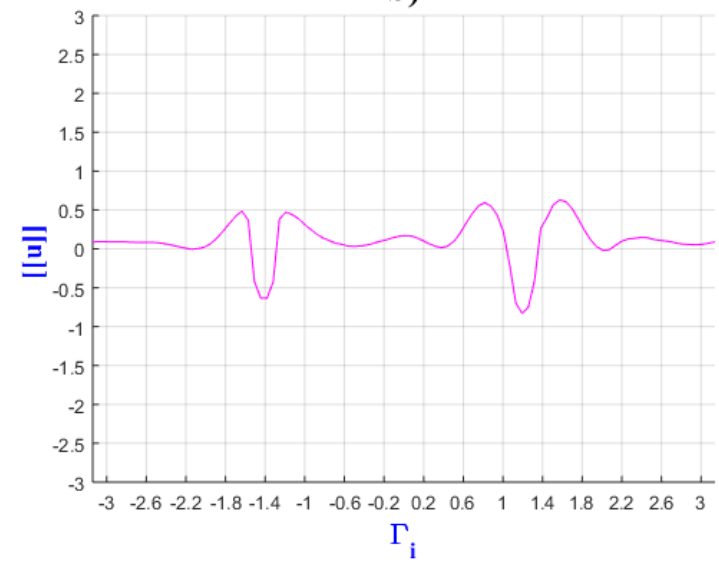

d)

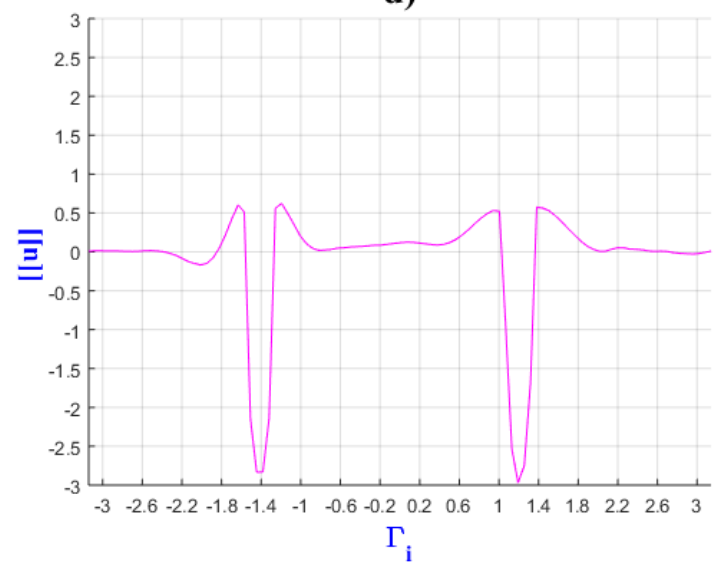

Fig. 10: Cracks with same size: Reconstructed jump $[[u]]=u^{+}-u^{-}$on $\Gamma_{i}$ for a test case with $\left.\left.\mathrm{t}=2 \mathrm{a}\right), \mathrm{b}\right) \mathrm{t}=4$, c) $\mathrm{t}=6$ and d) $\mathrm{t}=8, \xi=10^{-2}, \delta t=1$.

\begin{tabular}{|c|c|c|c|c|c|}
\hline $\begin{array}{c}\text { Number } \\
\text { of nodes } \\
\text { on } \Gamma_{c_{1}}\end{array}$ & $\begin{array}{c}\text { Number } \\
\text { of nodes } \\
\text { on } \Gamma_{c_{2}}\end{array}$ & $\begin{array}{c}\text { Number } \\
\text { of nodes } \\
\text { on } \Gamma_{i} \backslash \sigma\end{array}$ & $\begin{array}{c}\text { Number } \\
\text { of nodes } \\
\text { on } \boldsymbol{\sigma}\end{array}$ & $\begin{array}{c}\text { Total } \\
\text { number of } \\
\text { iterations }\end{array}$ & Figures \\
\hline 25 & 70 & 50 & 3 & 943 & Fig.13.a) \\
\hline 50 & 150 & 105 & 5 & 508 & Fig.13.b) \\
\hline 100 & 300 & 210 & 20 & 168 & Fig.13.c) \\
\hline 200 & 500 & 300 & 20 & 171 & Fig.13.d) \\
\hline
\end{tabular}

Table 1: Number of iteration as a function of the mesh size.

(P1) and $\gamma_{2}$ for problem (P2). The number of iterations needed are given in Table (2). Rather than producing more figures, we report here without illustrations that the reconstructions are sensitive with respect to the regularizing parameter. In our case, choosing the parameter such that $0.1 \leq \gamma_{1} \leq 1$ and $0.1 \leq \gamma_{2} \leq 0.8$ gives stable results improving with smaller values. A common ad-hoc way to choose the regularizing parameter is to compute the numerical solution for a range of values of this parameter decreasing to zero. Once unstable (oscillating) numerical results are produced, we know that the regularizing parameter has been chosen too small and no further improvement in terms of accuracy can be achieved. 
a)

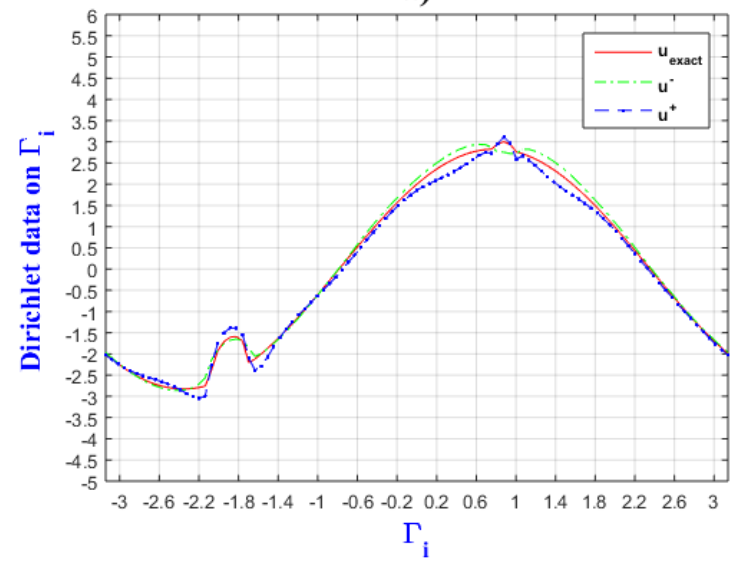

c)

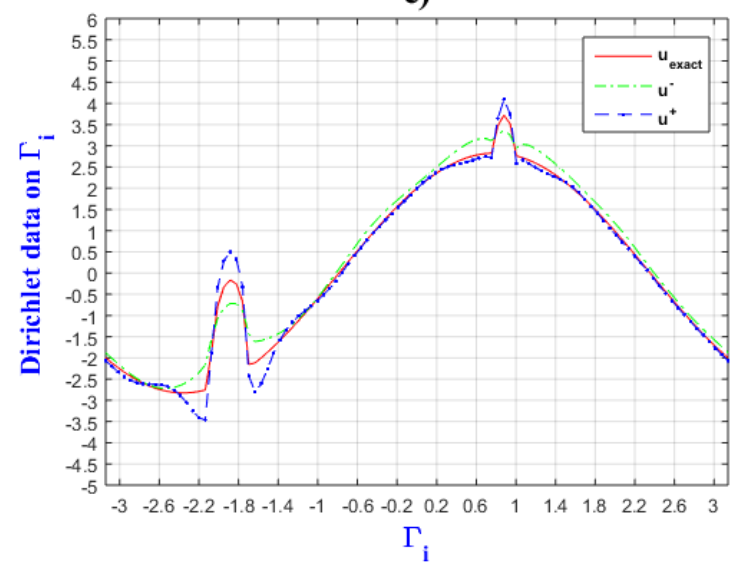

b)

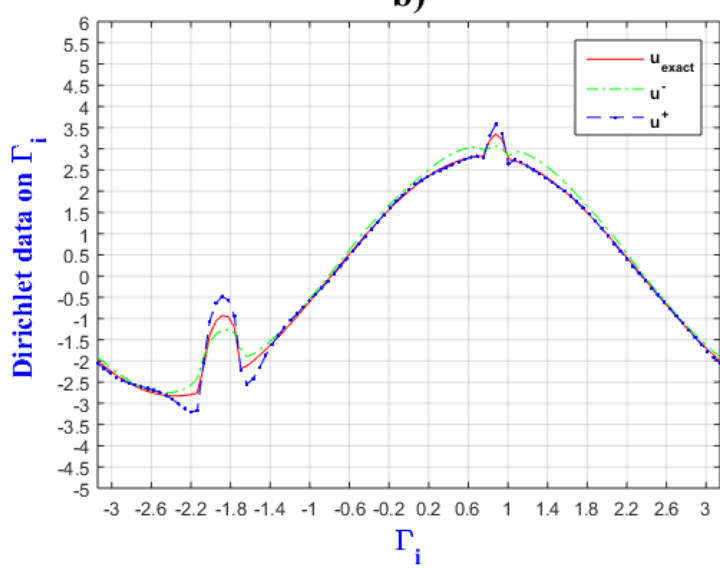

d)

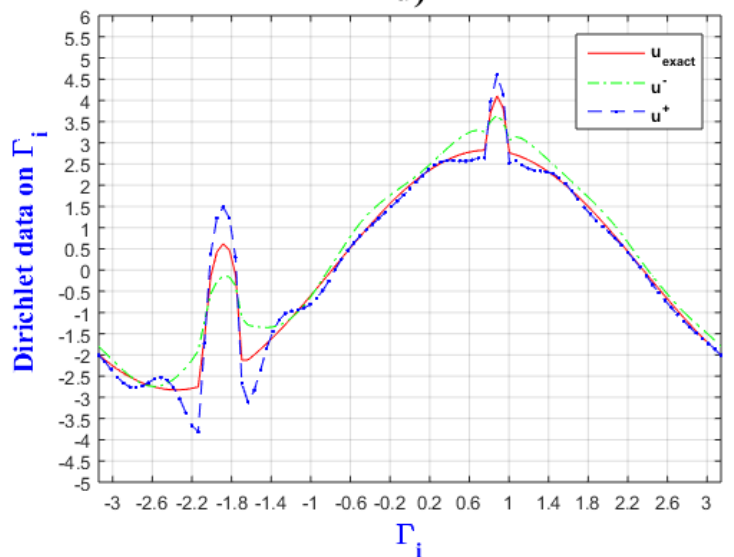

Fig. 11: Cracks of different size: Reconstruction of the Dirichlet date on $\Gamma_{i} u^{+}, u^{-}$and $u_{\text {exact }}$ for a test case with a) $\mathrm{t}=2 \mathrm{~b}$ ) $\mathrm{t}=4 \mathrm{c}) \mathrm{t}=6 \mathrm{~d}) \mathrm{t}=8, \xi=10^{-2}, \delta t=1$.

\begin{tabular}{|c||c|c|c|c|}
\hline$\gamma_{1}$ & 1 & 0.5 & 0.3 & 0.1 \\
\hline$\gamma_{2}$ & 0.8 & 0.4 & 0.3 & 0.1 \\
\hline Number of iterations & 168 & 334 & 465 & 1395 \\
\hline
\end{tabular}

Table 2: Number of iterations as a function of the regularization $\gamma$.

\subsection{Singular test case with noise}

To test the robustness of our procedure, we consider in the test case of singular crack the following noise of the boundary data $f$ :

$$
f=f+\psi \varepsilon,
$$

where $\psi$ denotes the noise level relative to $\|f\|_{L^{2}\left(\Gamma_{C}\right)}$, and $\varepsilon$ is a random function generated by the Freefeem++. 
a)

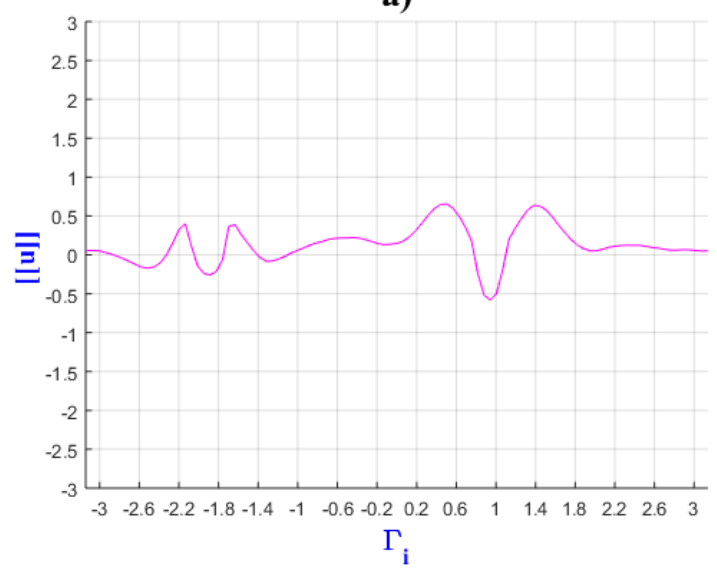

c)

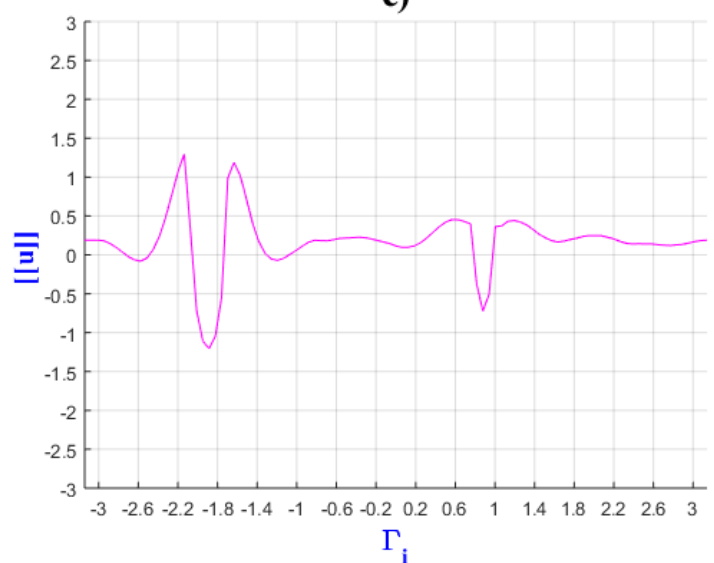

b)

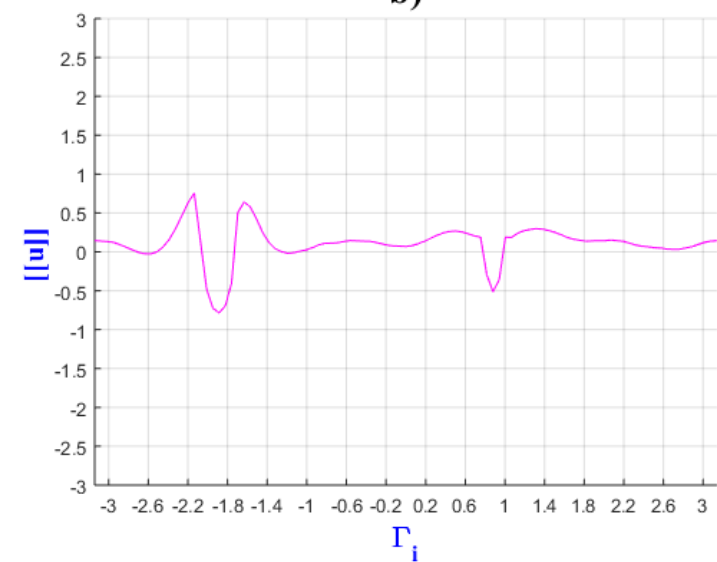

d)

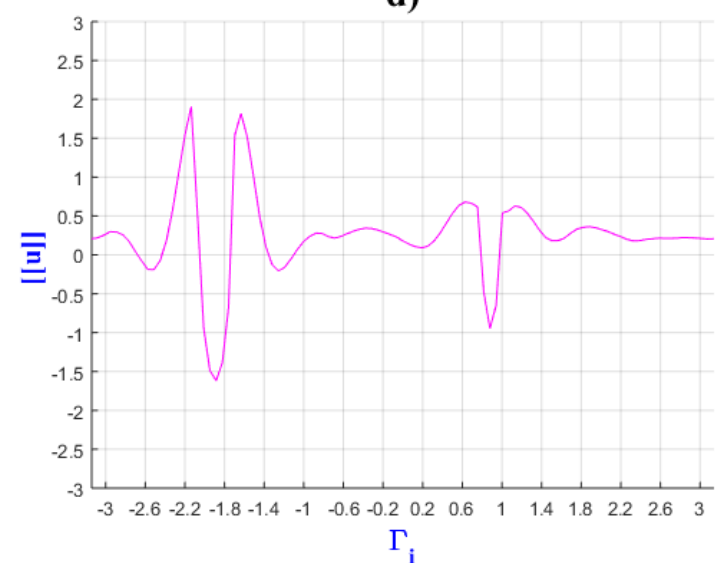

Fig. 12: Cracks of different size: Reconstructed jump $[[u]]=u^{+}-u^{-}$on $\Gamma_{i}$ for a test case with $\left.\mathrm{t}=2 \mathrm{a}\right)$, b) $\mathrm{t}=4$, c) $\mathrm{t}=6$ and d) $\mathrm{t}=8, \xi=10^{-2}, \delta t=1$.

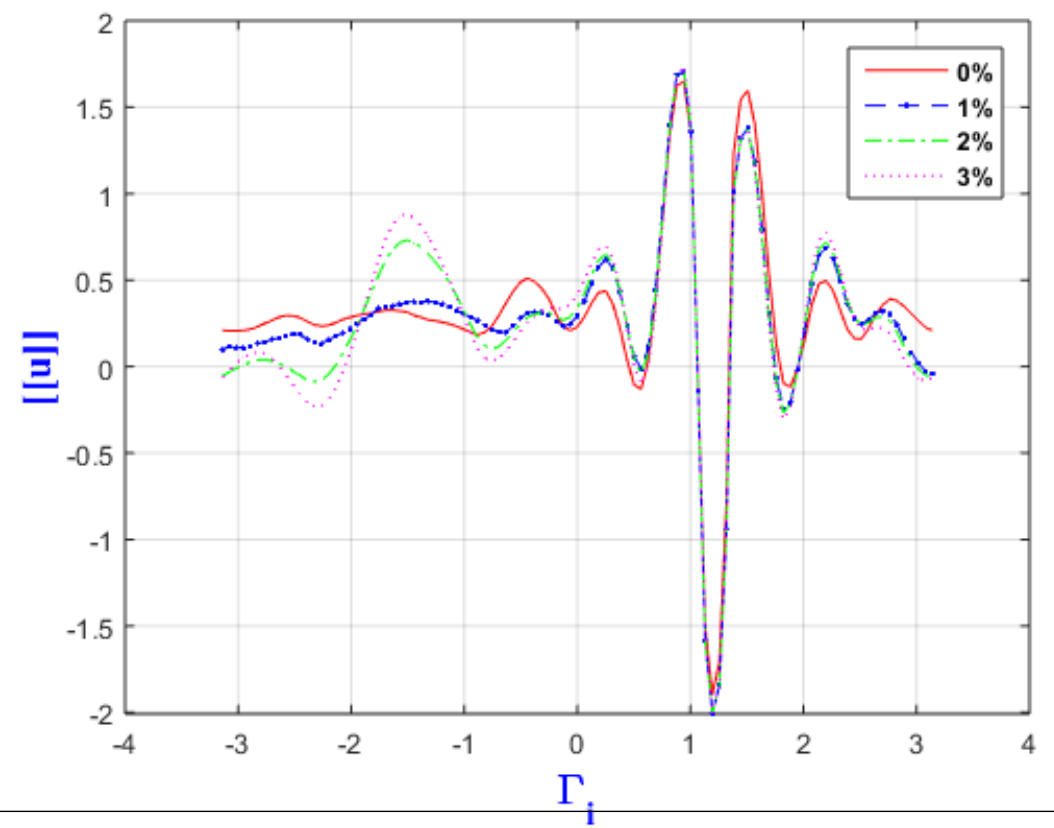

Fig. 14: Reconstructed jump with noisy data for the singular test case on $\Gamma_{i}$ at $\mathrm{t}=10$. 
a)

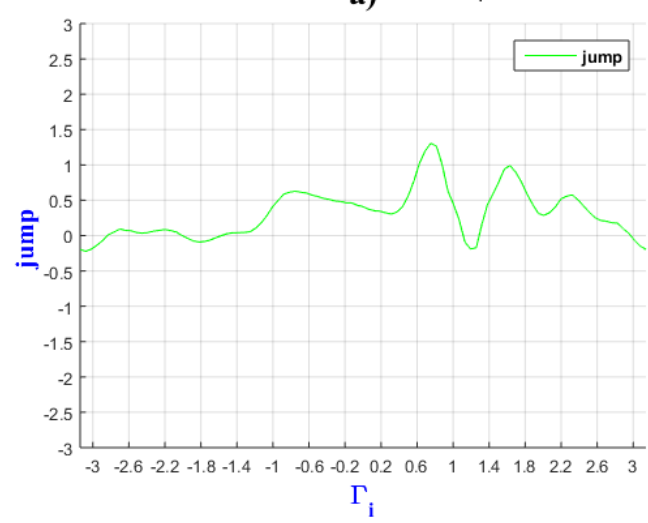

c)

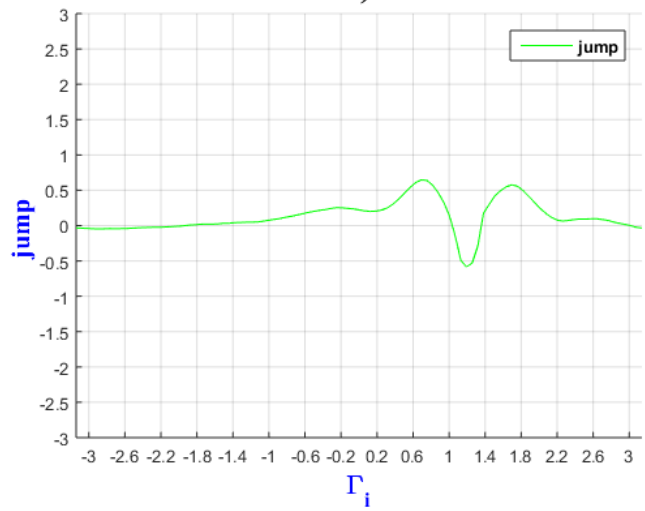

b)

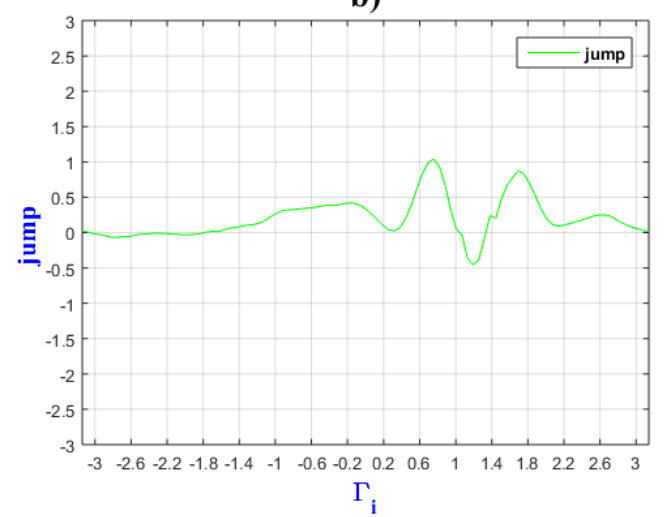

d)

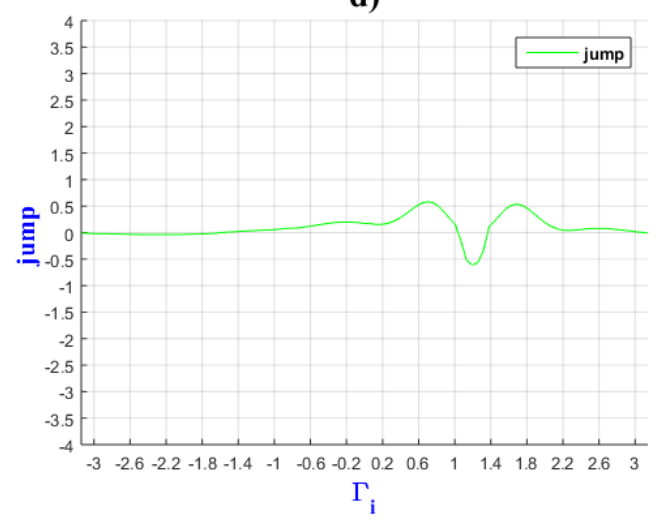

Fig. 13: Reconstructed jump $[[u]]=u^{+}-u^{-}$across $\Gamma_{i}$ with different types of mesh, $\xi=10^{-2}, \delta t=1$.

In Fig.14, we illustrate the results for various levels of noise at time $t=10$ where we plot the jump on $\Gamma_{i}$. The recovered jump with $1 \%$ is very close to the jump with $0 \%$. When the boundary data contains a higher level of noise i.e $2 \%$ and $3 \%$, the relative position of crack becomes less precise and finally the algorithm detects an incorrect number of cracks.

\section{Conclusion}

In this work, we have investigated the problem of identification of cracks. We proposed a strategy which transforms the problem into an alternative one which consists in solving two Cauchy problems. After that, we solved these Cauchy problems through the minimization of energy error functional.

The minimization of these functional leads to interfacial problems, which are solved by an iterative preconditioned gradient algorithm. Each gradient iteration requires to solve for each problem three well-posed problems. Numerical experiments illustrate the efficiency of the proposed method. The Recovered solutions are very close to the exact synthetic one, even for a singular test case. Moreover, the method does not require any regularization tool. Comparing the proposed method with classical least-square approaches and with other methods based on an energy norm would be an interesting future direction of investigation.

\section{Competing interests}

The authors declare that they have no competing interests. 


\section{Authors' contributions}

All authors have contributed to all parts of the article. All authors read and approved the final manuscript.

\section{References}

[1] G. Alessandrini and A. Diaz Valenzuela. Unique determination of multiple cracks by two measurements. SIAM J. Control Optim., 34(3):913-921, 1994.

[2] S. Andrieux and T. N. Baranger. Energy methods for Cauchy problems of evolutions equations. In Journal of Physics: Conference Series, volume 135, page 012007. IOP Publishing, 2008.

[3] S. Andrieux, T. N. Baranger, and A. Ben Abda. Solving Cauchy problems by minimizing an energy-like functional. Inverse problems, 22(1):115-133, 2006.

[4] S. Andrieux and A. Ben Abda. Identification of planar cracks by complete overdetermined data: inversion formulae. Inverse problems, 12(5):553-563, 1996.

[5] S. Andrieux, A. Ben Abda, and T. N. Baranger. Data completion via an energy error functional. Comptes Rendus Mécanique, 33(2):171-177, 2005.

[6] M. Azaïez, F. Ben Belgacem, and H. El Fekih. On Cauchy's problem: II. Completion, regularization and approximation. Inverse problems, 22(4):1307-1336, 2006.

[7] L. Baratchart, J. Leblond, F. Mandrea, and E. B. Saff. How can the meromorphic approximation help to solve some 2D inverse problems for the laplacian. Inverse Problems, 15(3):79-90, 1999.

[8] J. V. Beck, B. Blackwell, and Charles R. St. Clair Jr. Inverse heat conduction: Ill-posed problems. James Beck, 1985.

[9] A. Ben Abda and H. D. Bui. Planar crack identification for the transient heat equation. Inv. Ill-Posed Problems, 11(11):27-31, 2003.

[10] F. Ben Belgacem. Why is the Cauchy problem severely ill-posed? Inverse Problems, 23(2):823, 2007.

[11] F. Ben Belgacem and H. El Fekih. On Cauchy's problem: I. A variational Steklov-Poincaré theory. Inverse Problems, 21(6):19151936, 2005.

[12] M. Bertero, T. A. Poggio, and V. Torre. Ill-posed problems in early vision. Proceedings of the IEEE, 1988.

[13] A. Björck. Numerical Methods for Least Squares Problems. SIAM Journal on Mathematical Analysis, 1996.

[14] M. Brühl, M. Hanke, and M. Pidcock. Crack detection using electrostatic measurements. ESAIM: M2AN, 35(3):595-605, 2001.

[15] K. Bryan and M. Vogelius. A uniqueness result concerning the identification of a collection of cracks from nitely many elastostatic boundary measurements. SIAM J. Math. Anal., 23(4):950-958, 1992.

[16] A. Friedman and M. Vogelius. Determining cracks by boundary measurements. Indiana Univ. Math., 38:527-556, 1989.

[17] P. Grisvard. Singularities in boundary value problems, volume 22. Springer, 1992.

[18] J. Hadamard and Philip M. Morse. Lectures on Cauchy's problem in linear partial differential equations. Physics Today, 6:18, 1953.

[19] D. N. Hao and D Lesnic. The Cauchy problem for Laplaces equation via the conjugate gradient method. IMA Journal of Applied Mathematics, 65(2):199-217, 2000.

[20] F. Hecht, O. Pironneau, A. Le Hyaric, and K. Ohtsuka. Freefem++. Numerical Mathematics and Scientific Computation. Laboratoire J. L. Lions, Université Pierre et Marie Curie, 3, 2007.

[21] V. Isakov. Inverse Problems for Partial Differential Equations. Springer Science, 1998.

[22] S. Kowalevski. Zur theorie der partiellen difierentialgleichungen. J. Reine Angew. Math., 80:1-32, 1875.

[23] V. A. Kozlov, V. G. Maz'ya, and A. V. Fomin. An iterative method for solving the Cauchy problem for elliptic equations. Zhurnal Vychislitel'noi Matematiki i Matematicheskoi Fiziki, 31(1):64-74, 1991.

[24] P. Le Tallec, Y. H. De Roeck, and M. Vidrascu. Domain decomposition methods for large linearly elliptic three-dimensional problems. Journal of Computational and Applied Mathematics, 34(1):93-117, 1991.

[25] K. Miller. Least squares methods for ill-posed problems with a prescribed bound. SIAM Journal on Mathematical Analysis, 1(1):52-74, 1970.

[26] M. Moussaoui and B. K. Sadallah. Régularite des coefficients de propagation de singularités pour léquation de la chaleur dans un ouvert plan polygonal. CR Acad. Sci. Paris Ser. I Math, 293(5):297-300, 1981. 
[27] A. Quarteroni and A. Valli. Domain decomposition methods for partial differential equations. Oxford University Press, 1999.

[28] T. Wei, Y. C. Hon, and L. Ling. Method of fundamental solutions with regularization techniques for cauchy problems of elliptic operators. Engineering Analysis with Boundary Elements, 31(4):373-385, April 2007. 